\title{
Critical Current Problems in HTc Superconductors
}

\author{
J. SOSNOWSKI* \\ Electrotechnical Institute, M. Pożaryskiego 28, 04-703 Warsaw, Poland
}

\begin{abstract}
New results of the investigations critical current problems in the high temperature oxide superconductors are presented, basing on the flux pinning analysis. Model of the pinning interaction of the nanosized centers with pancake vortices is proposed, while comparison of the received results concerning the dependence of the potential barrier versus transport current with other pinning forces approaches is presented. Special attention is devoted to the investigations of the influence the initial position of the vortex captured at pinning center on the current-voltage characteristics and critical current.
\end{abstract}

PACS numbers: 74.72.-h, 74.25.Wx, 74.25.Sv, 74.25.Uv

\section{Introduction}

Critical current analysis of the high temperature oxide superconductors has great meaning both from the point of view of basic research as well as applications. New model of the interaction between pancake vortices and nanosized pinning centers has been elaborated. Existence of such nanodefects was observed in performed by us scanning electron microscopy (SEM) analysis of the structure of the HTc Bi:2223 tape, showing the microscopic defects in the form of the nanopores and unreacted $\mathrm{CuO}_{2}$ grains. Increase of the volume of the normal phase during movement of the captured vortex against the pinning center enhances the system energy and therefore its amount should be minimized. On the other hand, the Lorentz force acting on the pinned vortex during current flow, as well as elasticity forces tear off the vortices from the pinning centers, which leads then to their movement and dissipation effects. Potential barrier arises, being a function of these parameters: critical current density, pinning centers dimensions and as usually magnetic field and temperature. Critical current density for the flux creep process is reached, if the potential barrier height against tearing the vortices vanishes, while current filling the voltage criteria is achieved. This new model has been in the present paper elaborated especially taking into account various initial states of the pinned pancake vortices, as well as there has been performed comparison with other pinning forces models. Flux pinning was investigated previously in various papers mainly experimentally, see for instance [1-7].

\section{Results}

Capturing of the vortices on the crystalline defects is

\footnotetext{
* e-mail: sosnow@iel.waw.pl
}

an essential process from the point of view of the stabilization of the current flow through the superconducting materials. However, the physics of this process is further not explained up to the end. One of the questions is initial captured vortex position. In the previous paper [1] there has been considered the case of the vortex in the initial state captured onto the depth of the coherence length $\xi$ inside the pinning center. Such configuration can be treated as favorable because it should still retain unchanged pinned vortex structure due to the proximity effect, which allows to flow the screening current around the vortex core. Vortex shift against the pinning center enhances the normal state energy of the system composed from the pancake vortex captured on the pinning center. For evaluating the relevance of this initial configuration and better recognition of the model predictions for the final results describing the potential barrier height and current-voltage characteristics, the parallel calculations have been performed for other limiting initial vortex state. We have considered therefore additionally the configuration of the fully captured vortex, it is vortex core pinned on the depth of $2 \xi$ inside the pinning center of the nanosize. The basic equations describing the initial state normal energy for the fully captured pancake vortex as well as for vortex shifted against this initial position have been derived. Initial normal state energy of the system with the fully captured vortex, it is for $x=-\xi$, is equal to

$$
\begin{aligned}
& U(-\xi)=\frac{\mu_{0} H_{\mathrm{c}}^{2} l_{\mathrm{p}}}{2} \\
& \quad \times\left[\pi \xi^{2}-2 \xi^{2} \arcsin \frac{d}{2 \xi}-d \sqrt{\xi^{2}-\frac{d^{2}}{4}}\right] .
\end{aligned}
$$

$H_{\mathrm{c}}$ is the magnetic critical thermodynamic field, $l_{\mathrm{p}}$ nanosized pinning centre thickness, coherence length $\xi$ describes the radius of the pancake vortex core, while $d$ is the width of the nanosized pinning centre. The coordinate system has been chosen here in such a way that 
coordinate $x$ describes the distance between the middle of vortex core and pinning center edge. It means that $x=0$ corresponds to the half-captured vortex. For the first deflection of the pancake vortex from the initial position there appears the dependence

$$
\begin{aligned}
& U_{1}(x)=\frac{\mu_{0} H_{\mathrm{c}}^{2} l_{\mathrm{p}}}{2} \\
& \times\left[\pi \xi^{2}-2 \xi^{2} \arcsin \frac{d}{2 \xi}+\xi^{2} \arcsin \frac{\sqrt{\xi^{2}-x^{2}}}{\xi}\right. \\
& \left.-d \sqrt{\xi^{2}-\frac{d^{2}}{4}}+x \sqrt{\xi^{2}-x^{2}}\right]
\end{aligned}
$$

valid for the following range of the vortex deflection:

$$
-\xi \leq x \leq-\xi \sqrt{1-\left(\frac{d}{2 \xi}\right)^{2}} .
$$

For the next range of the vortex movement described by the boundary condition

$$
-\xi \sqrt{1-\left(\frac{d}{2 \xi}\right)^{2}} \leq x \leq \xi \sqrt{1-\left(\frac{d}{2 \xi}\right)^{2}}
$$

normal state energy of shifted vortex is given by the relation

$$
\begin{aligned}
& U_{2}(x)=\frac{\mu_{0} H_{\mathrm{c}}^{2} l_{\mathrm{p}} \xi^{2}}{2} \\
& \times\left[\pi-\arcsin \frac{d}{2 \xi}-\frac{d}{2 \xi} \sqrt{1-\left(\frac{d}{2 \xi}\right)^{2}}+d x\right] .
\end{aligned}
$$

For still larger pancake vortex shift against pinning center edge normal state potential is

$$
\begin{aligned}
& U_{3}(x)=\frac{\mu_{0} H_{\mathrm{c}}^{2} l_{\mathrm{p}} \xi^{2}}{2} \\
& \times\left[\frac{\pi}{2}+\arcsin \frac{x}{\xi}+\frac{x}{\xi} \sqrt{1-\left(\frac{x}{\xi}\right)^{2}}\right] .
\end{aligned}
$$

Equations (1)-(4) allow us to determine then the potential barrier height, which describes the currentvoltage characteristics and critical current. Transforming dependences (1)-(4) from translational into the current representation, for the case of the full vortex capturing, we obtain final relation describing the potential barrier height in the function of the current density reduced to the critical $i=j / j_{\mathrm{c}}$ :

$$
\begin{aligned}
& \Delta U(i)=\frac{\mu_{0} H_{\mathrm{c}}^{2} l_{\mathrm{p}} \xi^{2}}{2}\left[-\arcsin (i)-i\left(2+\sqrt{1-i^{2}}\right)\right. \\
& \left.+2 \arcsin \frac{d}{2 \xi}+\frac{d}{\xi} \sqrt{1-\left(\frac{d}{2 \xi}\right)^{2}}\right] .
\end{aligned}
$$

Equation (5) may be compared with the expression (6) describing the potential barrier height, received in the case of the half vortex capturing

$$
\begin{aligned}
& \Delta U(i)=\frac{\mu_{0} H_{\mathrm{c}}^{2} l_{\mathrm{p}} \xi^{2}}{2}\left[\arcsin \frac{d}{2 \xi}+\frac{d}{2 \xi} \sqrt{1-\left(\frac{d}{2 \xi}\right)^{2}}\right. \\
& \left.-i \sqrt{1-i^{2}}-\arcsin i\right] .
\end{aligned}
$$

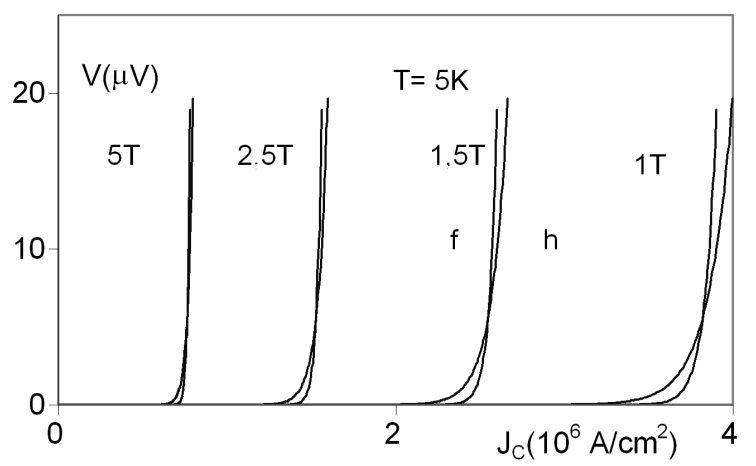

Fig. 1. Current-voltage characteristics for HTc superconductor, with (h) half-pinned pancake vortex in initial state, (f) fully-pinned vortex, for $d / 2 \xi=0.4$ versus applied external magnetic field.

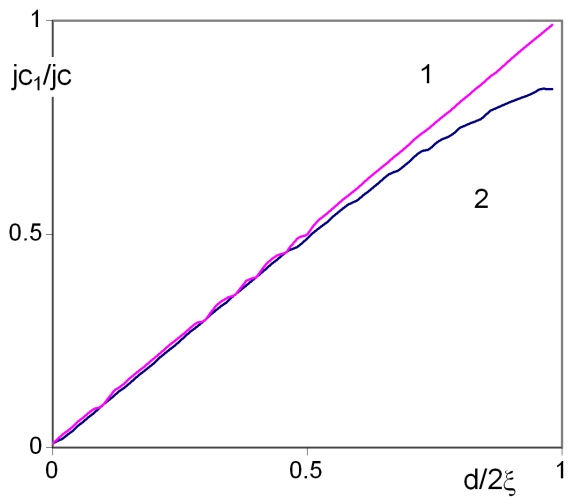

Fig. 2. The dependence of the reduced critical current density on the pinning center width $d$, for two initial positions of the pancake vortex: 1 - half-captured, 2

- fully-captured.

We insert then Eqs. (5), (6) into the constitutive relation describing the current-voltage characteristics in the flux creep process, in the function of the potential barrier height $\Delta U$. The results of the comparison of the calculations of the current-voltage characteristics for both (fully-captured and half-captured) initial states are shown in Fig. 1, for various magnetic fields. As follows from this figure for both cases of the fully captured vortex and for half-captured one the differences in the currentvoltage characteristics are at least in this case rather low. The dependence of the critical current on the pinning center width in reduced units for both initial positions of the captured vortex is presented in Fig. 2. Critical current density $j_{\mathrm{c} 1}$ shown in this figure is defined as the current density leading to the disappearance of the energy barrier $\Delta U$ in that case, while $j_{\mathrm{c}}$ is critical current density 


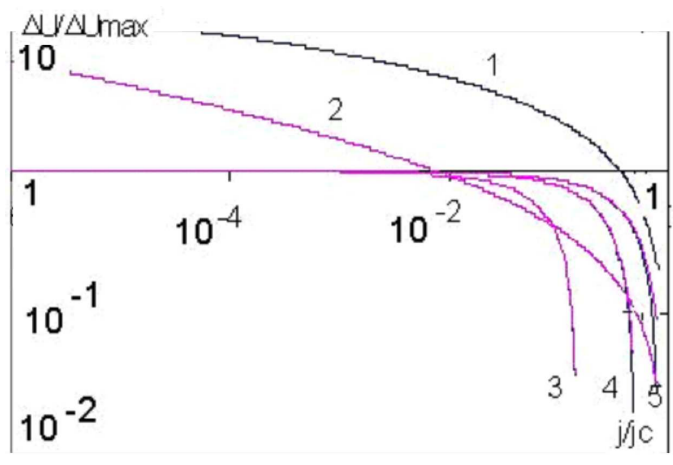

Fig. 3. Dependence of the reduced potential barrier on the reduced current normalized to the critical one for: (1) logarithmic dependence $\ln \left(j / j_{\mathrm{c}}\right)$ of the potential barrier, (2) power dependence of the form $\left(j / j_{\mathrm{c}}\right)^{-1 / 7}-1$, (3) in present model for $d / 2 \xi=0.15$, (4) $d / 2 \xi=0.5$ (5) $d / 2 \xi=0.95$.

in the limit of large size of the pinning center and half-captured initially vortex. Figure 3, on the other hand, presents the comparison of the reduced potential barrier in the function of the current normalized to the critical one for various pinning forces models $[2,3]$. The results obtained in the present approach are marked by numbers 3-5 and are in fact very similar for both limiting cases of the full and the half-full capturing vortices in the initial state. We should still consider the modification of the current distribution in the captured vortex, which can lead to the change of its shape. This electromagnetic effect is however beside the scope of this paper and will be regarded later.

\section{Conclusions}

Theoretical analysis of the critical current as well as critical temperature is further one of unresolved problems for the HTc superconductors. In the paper there is given a new approach to the critical current analysis. Special attention is concentrated on the subject of the influence of initial position of the captured vortex on the current-voltage characteristics. Two states have been considered of the half-captured and totally captured vortex. Results indicate that for both limiting cases critical $I-V$ curves and critical current are rather similar. There is an important conclusion showing that performed critical current analysis is not sensitive to this initial vortex position, which is not yet unequivocally determined.

\section{References}

[1] J. Sosnowski, Acta Phys. Pol. A 114, 269 (2008).

[2] M.V. Feigelman, V. Geshkenbein, A.I. Larkin, V. Vinokur, Phys. Rev. Lett. 63, 2303 (1983).

[3] A.I. Larkin, Yu.N. Ovchinnikov, J. Low Temp. Phys. 34, 409 (1979).

[4] H. Yamada, H. Yamasaki, K. Develos-Bagarinao, Y. Nakagawa, Y. Mawatari, J.C. Nie, H. Obara, S. Kosaka, Supercond. Sci. Technol. 17, 58 (2004).

[5] K. Okamura, M. Kiuchi, E.S. Otabe, T. Yasuda, T. Matsushita, S. Okayasu, Supercond. Sci. Technol. 17, S20 (2004).

[6] Y. Zhao, C.H. Cheng, Y. Feng, S. Shibata, N. Koshizuka, M. Murakami, Supercond. Sci. Technol. 17, S83 (2004).

[7] V.R. Misko, S. Savel'ev, A.L. Rakhmanov, F. Nori, Phys. Rev. B 75, 024509 (2007). 